\title{
No Existence of Black Holes at LHC Due to Minimal Length in Quantum Gravity
}

\author{
Ahmed Farag Ali \\ Dept. of Physics, Faculty of Sciences, Benha University, Benha 13518, Egypt. \\ ahmed.ali@fsc.bu.edu.eg ; ahmed.ali@uleth.ca
}

\begin{abstract}
We investigate the impact of the Generalized Uncertainty Principle (GUP), proposed by some approaches to quantum gravity such as String Theory and Doubly Special Relativity Theories (DSR) on the production of mini black holes, and show that the minimum black hole mass is formed at energies higher than the energy scales of LHC which possibly agrees with the recent experimental results of LHC $[1,2]$
\end{abstract}

\section{Introduction}

The existence of a minimal length is one of the most interesting predictions of some approaches related to quantum gravity such as String Theory as well as Black hole physics. This is a consequence of String Theory since strings can not interact at distances smaller than their size, which yields Generalized Uncertainty Principle (GUP) [3]. From Black hole physics, the Uncertainty Principle, $\Delta x \sim \hbar / \Delta p$, is modified at the Planck energy scale, when the corresponding Schwarzschild radius is comparable to the Compton wavelength (both are approximately equal to the Planck length). Higher energies result in a further increase of the Schwarzschild radius, resulting in $\Delta x \approx \ell_{P l}^{2} \Delta p / \hbar$ The above observation, along with a combination of thought experiments and rigorous derivations suggest that the Generalized Uncertainty Principle (GUP) holds at all scales, and is represented by [3-8]

$$
\begin{aligned}
\Delta x_{i} \Delta p_{i} & \geq \frac{\hbar}{2}\left[1+\beta\left((\Delta p)^{2}+<p>^{2}\right)\right. \\
& \left.+2 \beta\left(\Delta p_{i}^{2}+<p_{i}>^{2}\right)\right]
\end{aligned}
$$

where $p^{2}=\sum_{j} p_{j} p_{j}, \beta=\beta_{0} /\left(M_{p} c\right)^{2}=\beta_{0} \frac{\ell_{p}^{2}}{\hbar^{2}}, M_{p}=$ Planck mass, and $M_{p} c^{2}=$ Planck energy.

It was shown in [5], that inequality (1.1) is equivalent to the following modified Heisenberg algebra

$$
\left[x_{i}, p_{j}\right]=i \hbar\left(\delta_{i j}+\beta \delta_{i j} p^{2}+2 \beta p_{i} p_{j}\right) .
$$

This form ensures, via the Jacobi identity, that $\left[x_{i}, x_{j}\right]=0=\left[p_{i}, p_{j}\right][6]$.

Recently, we proposed the GUP in [10-12] which predicts maximum observable momenta besides the existence of minimal measurable length and is consistent with Doubly 
Special Relativity (DSR) theories, String Theory and Black Holes Physics and which ensures $\left[x_{i}, x_{j}\right]=0=\left[p_{i}, p_{j}\right]$ (via the Jacobi identity).

$$
\left[x_{i}, p_{j}\right]=i \hbar\left[\delta_{i j}-\alpha\left(p \delta_{i j}+\frac{p_{i} p_{j}}{p}\right)+\alpha^{2}\left(p^{2} \delta_{i j}+3 p_{i} p_{j}\right)\right]
$$

where $\alpha=\alpha_{0} / M_{p} c=\alpha_{0} \ell_{p} / \hbar, M_{p}=$ Planck mass, $\ell_{p}=$ Planck length, and $M_{p} c^{2}=$ Planck energy.

Note that Eq. (1.3) is approximately covariant under DSR transformations [13]. Since DSR transformations preserve both speed of light, and invariant energy scale [14], it is not surprising that Eqs. (1.3) imply the existence of minimum measurable length and maximum measurable momentum

$$
\begin{aligned}
& \Delta x \geq(\Delta x)_{\min } \approx \alpha_{0} \ell_{p} \\
& \Delta p \leq(\Delta p)_{\max } \approx \frac{M_{p} c}{\alpha_{0}} .
\end{aligned}
$$

Our proposed GUP suggests that the space is discrete [10-12] and that all measurable lengths are quantized in units of a fundamental minimum measurable length (which can be the Planck length). Note that similar quantization of length was shown in the context of Loop Quantum Gravity in [15].

Since the GUP modifies the fundamental commutator bracket between position and momentum, naturally it modifies the Hamiltonian and hence it affects a host of quantum phenomena, and it is important to make a quantitative study of these effects which would open a window for quantum gravity phenomenology. In a series of papers, the author with collaborators investigated the effects of GUP on atomic, condensed matter systems and preheating phase of the universe $[11,16-19]$. Also the author studied in [20] its effects on the weak equivalence principle (WEP) and the Liouville theorem (LT) in statistical mechanics, and it was found that the GUP can potentially explain the small observed violations of the WEP in neutron interferometry experiments [21] and also predicts a modified invariant phase space which is relevant to the Liouville theorem. Recently, it was suggested in [22] that the GUP can be measured directly in Quantum Optics Lab which confirm the theoretical predictions in $[16,18]$

The proposals for the existence of extra dimensions has opened up new doors of research in quantum gravity [23-26]. In particular, a host of interesting work is being done on different aspects of low-energy quantum gravity phenomenology. One of the most significant sub-fields is the study of black hole (BH) and brane production at the LHC [27].

In this paper, we present a phenomenological study of black holes in higher dimensions at the Large Hadron Collider (LHC) if GUP that follows from Jacobi Identity is taken into consideration, see Eqs. $(1.1,1.3)$. If the black hole can be produced and detected, it would result in an additional mass threshold above the Planck scale at which new physics can be found. The scope of the present work is to investigate the effect of GUP on the Hawking temperature, entropy, and $\mathrm{BH}$ decay rate. We find that the $\mathrm{BH}$ thermodynamics dramatically changed if the GUP parameter is non-vanishing.

We also obtain an interesting result that black holes may not be detectable at the current LHC energy scales. This result possibly agrees with the recent experiments that 
were done at LHC $[1,2]$, which tend to exclude the black hole observations in the current energy scales at LHC. The effect of the GUP on Black holes has been studied before with different versions of GUP which does not follow from Jacobi Identity, see e.g. [29], however the previous studies predicted that BH's can be seen at the LHC energy scales in disagreement with the recent experimental results of LHC [1,2]. The suppressed black hole production has been studied with with different approaches such as the non commutative geometry and minimal length theories [30].

An outline of this paper is as follows. In Sec .2, We introduce briefly how standard Hawking temperature can be obtained from the standard uncertainty principle in $D$ - dimensions. In Sec .3, we investigate BH thermodynamics if GUP of Eq. (1.1) which was proposed earlier by $[3-7]$ is taken into consideration. We found that the $\mathrm{BH}$ minimal mass could be detected in the range between $60 \mathrm{TeV}$ and $2.3 \times 10^{5} \mathrm{TeV}$ depending on the number of extra dimensions for $D=6 . .10$. In Sec. 4, we repeat the analysis for our proposed model of GUP in Eq. (1.3). We find that BH's minimal masses could be found in the energy scales between $13.8 \mathrm{TeV}$ and $5.5 \times 10^{3} \mathrm{TeV}$ for $D=6 . .10$. The results are explained in Sec. 5 . So, the threshold is less for the second case.

\section{Hawking Temperature - Uncertainty Relation Con- nection}

In this section, we review the connection between standard Hawking temperature and uncertainty relation that has been proposed by Adler et al. in [28] and has been generalized in large extra dimensions by Cavaglia et al in [29]. A BH could be modeled as $(D-1)$ dimensional sphere of size equal to twice of Schwarzschild radius, $r_{s}$. Since the Hawking radiation is a quantum process, so the emitted particle should obey the Heisenberg uncertainty relation. This leads to momentum-position uncertainty,

$$
\Delta p_{i} \Delta x_{j} \geq \frac{\hbar}{2} \delta_{i j}
$$

where the uncertainty in position of emitted Hawking particle has its minimum value given by

$$
\Delta x \approx 2 r_{s}=2 \lambda_{D}\left[\frac{G_{D} M}{c^{2}}\right]^{\frac{1}{D-3}},
$$

where $\lambda_{D}=\left[\frac{16 \pi}{(D-2) \Omega_{D-2}}\right]^{\frac{1}{D-3}}$ and $\Omega_{D}=\frac{2 \pi \frac{D-1}{2}}{\Gamma\left(\frac{D-1}{2}\right)}$.

Using Eq $(2.1,2.2)$ with the argument used in [29] that $\Delta x_{i} \Delta p_{i} \approx \Delta x \Delta p$, the energy uncertainty of the emitted Hawking particle is given by

$$
\Delta E \approx c \Delta p=c \frac{\hbar}{2 \Delta x} \approx c \frac{\hbar}{4 r_{s}}=\frac{M_{p} c^{2}}{4 \lambda_{D}}\left(\frac{M}{M_{p}}\right)^{\frac{-1}{(D-3)}} .
$$

From now on, we can assume $m=\frac{M}{M_{p}}$, where $m$ is the mass in units of the Planck mass and the Planck mass $M_{p}$ is given by $M_{p}=\left[\frac{\hbar^{D-3}}{c^{D-5} G_{D}}\right]^{\frac{1}{(D-2)}}$ in D-dimensions. As proposed 
by Adler et al. in [28], one can identify the energy uncertainty $\Delta E$ as the energy of the emitted photon from the black hole. Based on this argument, one can get the characteristic temperature of the emitted Hawking particle from the previous energy by just multiplying it with a calibration factor $\left(\frac{D-3}{\pi}\right)[28,29]$ to give exactly the Hawking temperature [31] in D-dimensions of the spacetime as follows:

$$
T_{H}=\frac{D-3}{4 \pi \lambda_{D}} M_{p} c^{2} m^{\frac{-1}{(D-3)}} .
$$

The thermodynamical properties of the $\mathrm{BH}$ can be computed via the usual thermodynamic relations. The entropy can be calculated using the first law of black hole thermodynamics,

$$
d M=\frac{1}{c^{2}} T d S .
$$

Using the mass in units of the Planck mass, $m$, one can rewrite Eq. (2.5) as follows:

$$
d S=M_{p} c^{2} \frac{1}{T} d m
$$

By integrating Eq. (2.6) using Eq. (2.4), one can obtain the the Bekenstein entropy [32] as follows

$$
S=\frac{4 \pi \lambda_{D}}{D-2} m^{(D-2) /(D-3)} .
$$

The specific heat can be calculated using the thermodynamical relation

$$
\mathcal{C}=T \frac{\partial S}{\partial T}=T \frac{\partial S}{\partial m} \frac{\partial m}{\partial T}=M_{p} c^{2} \frac{\partial m}{\partial T}
$$

where we have used Eq. (2.6) in the last equation.

By differentiating Eq. (2.4) and substituting this into Eq. (2.8), the specific heat could be given by

$$
\mathcal{C}=-4 \pi \lambda_{D} m^{\frac{(D-2)}{(D-3)}}
$$

The Hawking temperature $T_{H}$ can be used in the calculation of the emission rate. The emission rate might be calculated using Stefan-Botlzmann law if the energy loss was dominated by photons. Assuming $\mathcal{D}$-dimensional spacetime brane, the thermal emission in the bulk of the brane can be neglected and the black hole is supposed to radiate mainly on the brane [33], so the emission rate on the brane can be given by:

$$
\frac{d M}{d t} \propto T^{\mathcal{D}}
$$

Because the the black hole radiates mainly on the brane [33], i.e $\mathcal{D}=4$, the emission rate can be found as following:

$$
\frac{d m}{d t}=-\frac{\mu^{\prime}}{t_{p}} m^{\frac{-2}{(D-3)}}
$$


where $t_{p}=\left(\frac{\hbar G_{D}}{c^{D+1}}\right)^{\frac{1}{(D-2)}}$ is the Planck time, and the form of $\mu^{\prime}$ can be found in [29].

The decay time of the black hole can be obtained by integrating Eq. (2.11) to give

$$
\tau=\mu^{\prime-1}\left(\frac{D-3}{D-1}\right) m_{i}^{\frac{(D-1)}{(D-3)}} t_{p}
$$

Note that the calculated Hawking temperature $T_{H}$, Bekenstein entropy $S$, specific heat $\mathcal{C}$, emission rate $\frac{d m}{d t}$, and decay time $\tau$ lead to catastrophic evaporation as $m \rightarrow 0$. This can be explained as following. Since $\mathcal{C}=0$ only when $m=0$, the black hole will continue to radiate until $m=0$. But as the black hole approaches zero mass, its temperature approaches infinity with infinite radiation rate. This was just a brief summary for the Hawking radiation-Uncertainty principle connection, and the catastrophic implications of Hawking radiation as the black hole mass approaches zero. In the next two sections, we study BH thermodynamics if GUP is taken into consideration. The end-point of Hawking radiation is not catastrophic because GUP imply the existence of $\mathrm{BH}$ remnants at which the specific heat vanishes and, therefore, the BH cannot exchange heat with the surrounding space. The GUP prevents BHs from evaporating completely, just like the standard uncertainty principle prevents the hydrogen atom from collapsing [28, 29].

\section{$3 \quad$ GUP quadratic in $\Delta p$ and $\mathrm{BH}$ thermodynamics}

In this section, we make analysis of BH thermodynamics If GUP proposed in [3-7] is taken into consideration.

The emitted particles as Hawking radiation are mostly photons and standard model (SM) particles. According to the ADD model of extra dimensions [23], photons and SM particles are localized to the brane. So the photons or SM particles have mainly 4-components momentum and the other components in the extra dimensions are equal to zero. For simplicity, we might assume from kinetic theory of gases which assumes a cloud of points in velocity space, equally spread in all directions (there is no reason particle would prefer to be moving in the x-direction, say, rather than the y-direction) and consider:

$$
p_{1} \approx p_{2} \approx p_{3}
$$

This assumption leads to

$$
\begin{aligned}
p^{2} & =\sum_{i=1}^{3} p_{i} p_{i} \approx 3 p_{i}^{2} \\
\left\langle p_{i}^{2}\right\rangle & \approx \frac{1}{3}\left\langle p^{2}\right\rangle .
\end{aligned}
$$

So Eq. (1.1) reads, with using the argument used in [29],

$$
\Delta x \Delta p \geq \frac{\hbar}{2}\left[1+\frac{5}{3} \beta\left\langle p^{2}\right\rangle\right],
$$


Now, we want to find the relation between $\left\langle p^{2}\right\rangle$ and $\Delta p^{2}$. We can assume that we have a photon gas emitted from the BH like emission from a black body. Therefore, we might use Wien's Law which gives a temperature corresponding to a peak emission at energy given by

$$
c\langle p\rangle=2.821 T_{H},
$$

From Hawking-Uncertainty connection proposed by Adler et al. in [28] and that was generalized in large extra dimensions by Cavaglia et al in [29], we have

$$
T_{H}=\frac{D-3}{\pi} c \Delta p=\frac{1}{2.821} c\langle p\rangle .
$$

We get the following relations using the relation $\left\langle p^{2}\right\rangle=\Delta p^{2}+\langle p\rangle^{2}$

$$
\begin{aligned}
\langle p\rangle & =2.821 \frac{D-3}{\pi} \Delta p=\sqrt{\mu} \Delta p, \\
\left\langle p^{2}\right\rangle & =(1+\mu) \Delta p^{2}, \quad \text { where } \quad \mu=\left(2.821 \frac{D-3}{\pi}\right)^{2} .
\end{aligned}
$$

Using Eqs. $(3.2,3.6)$ in the inequality (3.3), we get

$$
\Delta x \Delta p \geq \frac{\hbar}{2}\left[1+\frac{5}{3}(1+\mu) \beta_{0} \ell_{p}^{2} \frac{\Delta p^{2}}{\hbar^{2}}\right] .
$$

By solving the inequality (3.7) as quadratic equation in $\Delta p$, we obtain

$$
\frac{\Delta p}{\hbar} \geq \frac{\Delta x}{\frac{5}{3}(1+\mu) \beta_{0} \ell_{p}^{2}}\left[1-\sqrt{1-\frac{\frac{5}{3}(1+\mu) \beta_{0} \ell_{p}^{2}}{\Delta x^{2}}}\right] .
$$

Where we considered only the negative sign(-) solution which gives the standard uncertainty relation as $\frac{\ell_{p}}{\Delta x} \rightarrow 0$.

Using the same arguments that were used in Sec. 2, the modified Hawking temperature will be given by:

$$
\begin{aligned}
& T_{H}^{\prime}=\frac{D-3}{\pi \beta_{0}} \frac{M_{p} c^{2}}{\frac{5}{\frac{5}{(1+\mu)}}} m^{\frac{1}{D-3}} \lambda_{D}\left[1-\sqrt{1-\frac{\frac{5}{3}(1+\mu) \beta_{0}}{4 \lambda_{D}^{2} m^{\frac{2}{D-3}}}}\right] . \\
& =2 T_{H}\left[1+\sqrt{1-\frac{\frac{5}{3}(1+\mu) \beta_{0}}{4 \lambda_{D}^{2} m^{\frac{2}{D-3}}}}\right]^{-1}
\end{aligned}
$$

The modified Hawking temperature is physical as far as the black hole mass satisfies the following inequality:

$$
4 \lambda_{D}^{2} m^{\frac{2}{D-3}} \geq \frac{5}{3}(1+\mu) \beta_{0}
$$


This tells us the black hole should have minimum mass $M_{\min }$ given by

$$
M_{\text {min }}=M_{p}\left(\sqrt{\frac{\frac{5}{3}(1+\mu)}{4}}\right)^{D-3} \frac{D-2}{8 \Gamma\left(\frac{D-1}{2}\right)}\left(\sqrt{\beta_{0}} \sqrt{\pi}\right)^{D-3} .
$$

Here we note the minimum mass of the $\mathrm{BH}$ is different from the one obtained in [29]. There is a new factor $(\sqrt{5(1+\mu) / 12})^{D-3}$ which would give higher values for the minimum mass of the BHs. This factor appeared because we considered the GUP that follows from Jacobi identity (see 1.1) [6] and which Cavaglia et al. [29] did not consider.

The endpoint of Hawking evaporation in the GUP-case is characterized by a Planck-size remnant with maximum temperature

$$
T_{\max }=2 T_{H} .
$$

The emission rate can be calculated using Stefan-Boltzmann Law, using Eq. (2.10,2.11), Since the BH mostly emitting on the brane, so we consider 4-dimensional brane, so we get

$$
\frac{d m}{d t}=-16 \frac{\mu^{\prime}}{t_{p}} m^{\frac{-2}{D-3}}\left[1+\sqrt{1-\frac{\frac{5}{3}(1+\mu) \beta_{0}}{4 \lambda_{D}^{2} m^{\frac{2}{D-3}}}}\right]^{-4}
$$

The entropy can be calculated from the first law of BH-thermodynamics,

$$
d S=\frac{2 \pi}{D-3} \lambda_{D} m^{\frac{1}{D-3}}\left[1+\sqrt{1-\frac{\frac{5}{3}(1+\mu) \beta_{0}}{4 \lambda_{D}^{2} m^{\frac{2}{D-3}}}}\right] d m .
$$

The specific heat has been calculated in GUP-case to give

$\mathcal{C} \equiv T \frac{\partial S}{\partial T}=M_{p} c^{2} \frac{\partial m}{\partial T}=-2 \pi \lambda_{d} m^{(d-2) /(d-3)} \sqrt{1-\frac{\frac{5}{3}(1+\mu) \beta_{0}}{4 \lambda_{D}^{2} m^{\frac{2}{D-3}}}}\left(1+\sqrt{1-\frac{\frac{5}{3}(1+\mu) \beta_{0}}{4 \lambda_{D}^{2} m^{\frac{2}{D-3}}}}\right)$.

We note the $\mathrm{BH}$ specific heat vanishes at the minimum BH-mass. Therefore, the $\mathrm{BH}$ cannot exchange heat with the surrounding space. This may solve the problem of catastrophic evaporation of the $\mathrm{BH}$ that was discussed in the previous section.

\section{GUP linear and quadratic in $\Delta p$ and $\mathrm{BH}$ thermo- dynamics}

In this section, we would like to find the corresponding inequality for Eq. (1.3) in $(D-1)$ dimensions. Eq. (1.3) gives with using the argument used in [29],

$$
\Delta x \Delta p \geq \frac{\hbar}{2}\left[1-\alpha\langle p\rangle-\alpha\left\langle\frac{p_{i}^{2}}{p}\right\rangle+\alpha^{2}\left\langle p^{2}\right\rangle+3 \alpha^{2}\left\langle p_{i}^{2}\right\rangle\right] .
$$

Using arguments in the Sec. 3, Eqs. $(3.2,3.6)$, in the inequality (4.1), we get 


$$
\Delta x \Delta p \geq \frac{\hbar}{2}\left[1-\alpha_{0} \ell_{p}\left(\frac{4}{3}\right) \sqrt{\mu} \frac{\Delta p}{\hbar}+2(1+\mu) \alpha_{0}^{2} \ell_{p}^{2} \frac{\Delta p^{2}}{\hbar^{2}}\right] .
$$

The last inequality is ( and as far as we know the only one) following from Eq. (1.3).

By solving the inequality (4.2) as quadratic equation in $\Delta p$, we obtain

$$
\frac{\Delta p}{\hbar} \geq \frac{2 \Delta x+\alpha_{0} \ell_{p}\left(\frac{4}{3} \sqrt{\mu}\right)}{4(1+\mu) \alpha_{0}^{2} \ell_{p}^{2}}\left[1-\sqrt{1-\frac{8(1+\mu) \alpha_{0}^{2} \ell_{p}^{2}}{\left(2 \Delta x+\alpha_{0} \ell_{p}\left(\frac{4}{3}\right) \sqrt{\mu}\right)^{2}}}\right] .
$$

Where we considered only the negative $\operatorname{sign}(-)$ solution which gives the standard uncertainty relation as $\frac{\ell_{p}}{\Delta x} \rightarrow 0$.

Using the same arguments that were used in Sec. 2, the modified Hawking temperature will be given by:

$$
\begin{aligned}
T_{H}^{\prime} & =\frac{D-3}{\pi \alpha_{0}^{2}} \frac{M_{p} c^{2}}{(1+\mu)}\left(m^{\frac{1}{D-3}} \lambda_{D}+\frac{\alpha_{0} \sqrt{\mu}}{3}\right)\left[1-\sqrt{1-\frac{(1+\mu) \alpha_{0}^{2}}{2\left(\lambda_{D} m^{\frac{1}{D-3}}+\frac{\alpha_{0} \sqrt{\mu}}{3}\right)^{2}}}\right] \\
& =2 T_{H}\left(1+\frac{\alpha_{0} \sqrt{\mu}}{3 \lambda_{D} m^{\frac{1}{D-3}}}\right)^{-1}\left[1+\sqrt{1-\frac{(1+\mu) \alpha_{0}^{2}}{2\left(\lambda_{D} m^{\frac{1}{D-3}}+\frac{\alpha_{0} \sqrt{\mu}}{3}\right)^{2}}}\right]^{-1}
\end{aligned}
$$

The modified Hawking temperature is physical as far as the black hole mass satisfies the following inequality:

$$
(1+\mu) \alpha_{0}^{2} \leq 2\left(\lambda_{D} m^{\frac{1}{D-3}}+\frac{\alpha_{0} \sqrt{\mu}}{3}\right)^{2}
$$

This tells us the black hole should have minimum mass $M_{\min }$ given by

$$
M_{\text {min }}=M_{p}\left(\sqrt{\frac{(1+\mu)}{2}}-\sqrt{\frac{\mu}{9}}\right)^{D-3} \frac{D-2}{8 \Gamma\left(\frac{D-1}{2}\right)}\left(\alpha_{0} \sqrt{\pi}\right)^{D-3} .
$$

Here we note the minimum mass of the $\mathrm{BH}$ is different from the one obtained in [29]. There is a new factor $(\sqrt{(1+\mu) / 2}-\sqrt{\mu / 9})^{D-3}$ which would give higher values for the minimum mass of the BHs. This factor appeared because we considered our proposed GUP in [10-12] that follows from Jacobi Identity (see 1.3) [10-12].

The endpoint of Hawking evaporation in the GUP-case is characterized by a Planck-size remnant with maximum temperature

$$
T_{\text {max }} \approx 2\left[\frac{\frac{3(1+\mu)}{2}+\sqrt{\frac{\mu(\mu+1)}{2}}}{\frac{3}{2}+\frac{7}{6} \mu}\right] T_{H} .
$$

The emission rate can be calculated using Stefan-Boltzmann Law, using Eq. (2.10,2.11), we get for 4-dimensional brane: 


$$
\frac{d m}{d t}=-16 \frac{\mu^{\prime}}{t_{p}} m^{\frac{-2}{D-3}}\left(1+\frac{\alpha_{0} \sqrt{\mu}}{3 \lambda_{D} m^{\frac{1}{D-3}}}\right)^{-4}\left[1+\sqrt{1-\frac{(1+\mu) \alpha_{0}^{2}}{2\left(\lambda_{D} m^{\frac{1}{D-3}}+\frac{\alpha_{0} \sqrt{\mu}}{3}\right)^{2}}}\right]^{-4}
$$

The entropy can be calculated from the first law of BH-thermodynamics,

$$
d S=\frac{2 \pi}{D-3} \lambda_{D} m^{\frac{1}{D-3}}\left(1+\frac{\alpha_{0} \sqrt{\mu}}{3 \lambda_{D} m^{\frac{1}{D-3}}}\right)\left[1+\sqrt{1-\frac{(1+\mu) \alpha_{0}^{2}}{2\left(\lambda_{D} m^{\frac{1}{D-3}}+\frac{\alpha_{0} \sqrt{\mu}}{3}\right)^{2}}}\right] d m .
$$

The specific heat has been calculated in GUP-case to give

$$
\begin{aligned}
\mathcal{C}= & -\frac{2 \pi}{\lambda_{D}} m^{\frac{D-4}{D-3}}\left(\lambda_{D} m^{\frac{1}{D-3}}+\frac{\alpha_{0} \sqrt{\mu}}{3}\right)^{2} \sqrt{1-\frac{(1+\mu) \alpha_{0}^{2}}{2\left(\lambda_{D} m^{\frac{1}{D-3}}+\frac{\alpha_{0} \sqrt{\mu}}{3}\right)^{2}}} \\
& {\left[1+\sqrt{1-\frac{(1+\mu) \alpha_{0}^{2}}{2\left(\lambda_{D} m^{\frac{1}{D-3}}+\frac{\alpha_{0} \sqrt{\mu}}{3}\right)^{2}}}\right] . }
\end{aligned}
$$

We note the $\mathrm{BH}$ specific heat vanishes at the minimum $\mathrm{BH}$-mass. Therefore, the $\mathrm{BH}$ cannot exchange heat with the surrounding space.

\section{NO black holes at LHC current energy scales due to GUP}

In this section, we use the calculations in Sec. 3, and Sec. 4 to investigate whether black holes could be formed at LHC energy scales. From Eqs. ( 3.12, 4.7 ), we note that black holes can be formed with masses larger than $M_{p}$ in $D$-dimensions. The model of GUPblack holes in higher dimensions has three unknown parameters: $D, M_{P}$, and $\beta_{0}\left(\alpha_{0}\right)$. If we fix the GUP-parameters to be $\beta_{0}=1\left(\alpha_{0}=1\right)$. In this case, the values for the minimum black hole masses in the extra dimensions, using Eqs. $(3.12,4.7)$, are shown in the following Table.

In table 1 , a $\mathrm{BH}$ in $D$-dimensions at fixed $\beta_{0}=1$ can form only for energies equal to or larger than its minimum mass. We consider the latest observed limits on the ADD model parameter $M_{p}$ in [34].

This means BH's ( If GUP-quadratic in $\Delta p$ is only considered) in $D=6$ can form only at energies not less than $60.7 \mathrm{TeV}$, and for $D=8$, they can form only for energies not less than $2714 \mathrm{TeV}$, and for BH's in $D=10$, they can only form for energies not less than $2.3 \times 10^{5} \mathrm{TeV}$.

Turning to GUP-linear and quadratic in $\Delta p$ case, we found that the black hole can be formed at energies less than the ones predicted by GUP-quadratic case, but they are still 
Table 1: BH minimal mass for different dimensions using the latest observed limits on the ADD model parameter $M_{p}$ in [34].

\begin{tabular}{|c|c|c|c|c|}
\hline & & GUP-Quadratic: $\beta_{0}=1$ & GUP-Linear\&Quadratic: $\alpha_{0}=1$ & GUP-Quadratic of [29] \\
\hline \hline$D$ & $M_{p}$ & $M_{\min }$ & $M_{\min }$ & $M_{\min }$ \\
\hline 6 & $4.54 \mathrm{TeV}$ & $>60.7 \mathrm{TeV}$ & $>13.8 \mathrm{TeV}$ & $>2.1 \mathrm{TeV}$ \\
\hline 7 & $3.51 \mathrm{TeV}$ & $>362 \mathrm{TeV}$ & $>46.3 \mathrm{TeV}$ & $>3.1 \mathrm{TeV}$ \\
\hline 8 & $2.98 \mathrm{TeV}$ & $>2714 \mathrm{TeV}$ & $>196 \mathrm{TeV}$ & $>3.9 \mathrm{TeV}$ \\
\hline 9 & $2.71 \mathrm{TeV}$ & $>24 \times 10^{3} \mathrm{TeV}$ & $>982 \mathrm{TeV}$ & $>4.5 \mathrm{TeV}$ \\
\hline 10 & $2.51 \mathrm{TeV}$ & $>2.3 \times 10^{5} \mathrm{TeV}$ & $>5.5 \times 10^{3} \mathrm{TeV}$ & $>4.7 \mathrm{TeV}$ \\
\hline
\end{tabular}

Table 2: The Schwarzschild radius $R_{s}=\frac{1}{\sqrt{\pi} M_{p}}\left[\frac{M_{B H}}{M_{p}} \frac{8 \Gamma\left(\frac{D-1}{2}\right)}{D-2}\right]^{\frac{1}{D-3}}$ using the latest observed limits on the ADD model parameter $M_{p}$ in [34].

\begin{tabular}{|c|c|c|c|}
\hline & GUP-Quadratic: $\beta_{0}=1$ & GUP-Linear\&Quadratic: $\alpha_{0}=1$ & GUP-Quadratic of [29] \\
\hline \hline$D$ & $R_{s}$ & $R_{s}$ & $R_{s}$ \\
\hline 6 & $>0.41$ & $>0.25$ & $>1$ \\
\hline 7 & $>0.69$ & $>0.41$ & $>0.99$ \\
\hline 8 & $>0.99$ & $>0.58$ & $>0.99$ \\
\hline 9 & $>1.31$ & $>0.77$ & $>1$ \\
\hline 10 & $>1.64$ & $>0.96$ & $>0.99$ \\
\hline
\end{tabular}

larger than the current energy scales of LHC. The BH's in $D=6$ can form only at energies not less than $13.8 \mathrm{TeV}$, and BH's can form in $D=7$ for energies not less than $46.3 \mathrm{TeV}$.

In the table 1, we compare our results with the results proposed in [29]. The previous studies in [29] predicted that BH's might be seen at the energy scales of LHC in disagreement with the recent experimental results of LHC [1,2]. Our results possibly agrees with the results of the experiment $[1,2]$. We found that black holes can be formed at energies much higher than the current energy scales of LHC. Predictions of mini black holes forming at collision energies of a few TeV's were based on theories that consider the gravitational effects of extra dimensions of space like string theory and large extra dimensions theories [23-27]. But scientists at the Compact Muon Solenoid (CMS) detector in LHC are excluding semiclassical and quantum black holes with masses below 3.8 to 5.3 TeV. Our proposed model of GUP can possibly justify why higher energies larger than the current scale of LHC is needed to form mini black holes. 


\section{Conclusions}

We investigated whether the GUP can explain the formation of black holes at energies higher than the energy scales of LHC to explain the recent experimental results that were obtained at LHC $[1,2]$. We have shown that, by studying Hawking-Uncertainty connection, the black holes can be formed in the range between $13.8-5.5 \times 10^{3} \mathrm{TeV}$ for GUP-linear and quadratic in $\Delta p$ for values of $D$ between 6 and 10, and they can be formed in the range between $60-2.3 \times 10^{5} \mathrm{TeV}$ for GUP-quadratic in $\Delta p$ for values of $D$ between 6 and 10. Both cases say black holes can be formed at energies higher than the current energy scales of LHC. We conclude that mechanisms such as GUP may be necessary to explain the recent experimental results. In the future, it would be appropriate to apply our approach on the calculations of the cosmological constant, black body radiation, etc. We hope to report on these in the future.

\section{Acknowledgments ;}

The author gratefully thanks Saurya Das for many enlightening discussions and comments to finish this paper. The author gratefully thanks the anonymous referee for useful comments and suggestions which helped to improve the paper.

\section{References}

[1] V. Khachatryan et al. [CMS Collaboration], Phys. Lett. B 697, 434 (2011) [arXiv:1012.3375 [hep-ex]].

[2] S. Chatrchyan et al. [CMS Collaboration], JHEP 1204, 061 (2012) [arXiv:1202.6396 [hep-ex]].

[3] D. Amati, M. Ciafaloni, G. Veneziano, Phys. Lett. B 216 (1989) 41;

[4] M. Maggiore, Phys. Lett. B 304 (1993) 65 [arXiv:hep-th/9301067]; M. Maggiore, Phys. Rev. D 49 (1994) 5182 [arXiv:hep-th/9305163]; M. Maggiore, Phys. Lett. B 319 (1993) 83 [arXiv:hep-th/9309034]; L. J. Garay, Int. J. Mod. Phys. A 10 (1995) 145 [arXiv:gr-qc/9403008]; F. Scardigli, Phys. Lett. B 452 (1999) 39 [arXiv:hepth/9904025]; S. Hossenfelder, M. Bleicher, S. Hofmann, J. Ruppert, S. Scherer and H. Stoecker, Phys. Lett. B 575 (2003) 85 [arXiv:hep-th/0305262]; C. Bambi and F. R. Urban, Class. Quant. Grav. 25 (2008) 095006 [arXiv:0709.1965 [gr-qc]].

[5] A. Kempf, G. Mangano, R. B. Mann, Phys. Rev. D52 (1995) 1108 [arXiv:hepth/9412167].

[6] A. Kempf, J.Phys. A 30 (1997) 2093 [arXiv:hep-th/9604045].

[7] F. Brau, J. Phys. A 32 (1999) 7691 [arXiv:quant-ph/9905033].

[8] S. Hossenfelder, arXiv:1203.6191 [gr-qc]. 
[9] A. Kempf, Phys. Rev. D 54, 5174 (1996) [Erratum-ibid. D 55, 1114 (1997)] [arXiv:hepth/9602119]; A. Kempf and G. Mangano, Phys. Rev. D 55 (1997) 7909 [arXiv:hepth/9612084]; A. Kempf, arXiv:hep-th/9612082.

[10] A. F. Ali, S. Das and E. C. Vagenas, Phys. Lett. B 678, 497 (2009) [arXiv:0906.5396 [hep-th]],

[11] A. F. Ali, S. Das and E. C. Vagenas, arXiv:1001.2642 [hep-th].

[12] S. Das, E. C. Vagenas and A. F. Ali, Phys. Lett. B 690, 407 (2010) [arXiv:1005.3368 [hep-th]].

[13] J. L. Cortes, J. Gamboa, Phys. Rev. D71 (2005) 065015 [arXiv:hep-th/0405285];

[14] J. Magueijo and L. Smolin, Phys. Rev. Lett. 88 (2002) 190403 [arXiv:hep-th/0112090]; J. Magueijo and L. Smolin, Phys. Rev. D 71 (2005) 026010 [arXiv:hep-th/0401087].

[15] T. Thiemann, J. Math. Phys. 39 (1998) 3372-3392 [arXiv:gr-qc/9606092].

[16] S. Das, E. C. Vagenas, Phys. Rev. Lett. 101 (2008) 221301 [arXiv:0810.5333 [hep-th]].

[17] S. Das, E. C. Vagenas, Can. J. Phys. 87 (2009) 233 [arXiv:0901.1768 [hep-th]].

[18] A. F. Ali, S. Das and E. C. Vagenas, Phys. Rev. D 84, 044013 (2011) [arXiv:1107.3164 [hep-th]].

[19] W. Chemissany, S. Das, A. F. Ali and E. C. Vagenas, JCAP 1112, 017 (2011) [arXiv:1111.7288 [hep-th]].

[20] A. F. Ali, Class. Quant. Grav. 28, 065013 (2011) [arXiv:1101.4181 [hep-th]]

[21] R. Collela, A. W. Overhauser, and S. A. Werner, Phys. Rev. Lett. 34, 1472 (1975); K. C. Littrell, B. E. Allman, and S. A. Werner, Phys. Rev. A 56, 1767 (1997); A. Camacho and A. Camacho-Galvan, Reports on Progress in Physics 70 (2007), pages 1-56. arXiv:0810.1325 [gr-qc].

[22] I. Pikovski, M. R. Vanner, M. Aspelmeyer, M. Kim, C. Brukner, M. S. Kim and C. Brukner, Nature Phys. 8, 393 (2012) [arXiv:1111.1979 [quant-ph]].

[23] N. Arkani-Hamed, S. Dimopoulos and G. R. Dvali, Phys. Lett. B 429, 263 (1998) [arXiv:hep-ph/9803315].

[24] I. Antoniadis, N. Arkani-Hamed, S. Dimopoulos et al., Phys. Lett. B436, 257-263 (1998). [hep-ph/9804398].

[25] L. Randall, R. Sundrum, Phys. Rev. Lett. 83, 3370-3373 (1999). [hep-ph/9905221].

[26] L. Randall, R. Sundrum, Phys. Rev. Lett. 83, 4690-4693 (1999). [hep-th/9906064]. 
[27] S. Dimopoulos, G. L. Landsberg, Phys. Rev. Lett. 87, 161602 (2001). [hepph/0106295]; S. B. Giddings and S. D. Thomas, Phys. Rev. D 65, 056010 (2002) [hep-ph/0106219]; T. Banks and W. Fischler, hep-th/9906038.

[28] R. J. Adler, P. Chen, D. I. Santiago, Gen. Rel. Grav. 33, 2101-2108 (2001). [grqc/0106080].

[29] M. Cavaglia, S. Das, R. Maartens, Class. Quant. Grav. 20, L205-L212 (2003). [hepph/0305223]; M. Cavaglia, S. Das, Class. Quant. Grav. 21, 4511-4522 (2004). [hepth/0404050]; A. J. M. Medved, E. C. Vagenas, Phys. Rev. D70, 124021 (2004). [hepth/0411022].

[30] S. Hossenfelder, Phys. Lett. B598, 92-98 (2004). [hep-th/0404232]; P. Nicolini, Int. J. Mod. Phys. A 24, 1229 (2009) [arXiv:0807.1939 [hep-th]]; M. Sprenger, P. Nicolini and M. Bleicher, Eur. J. Phys. 33, 853 (2012) [arXiv:1202.1500 [physics.ed-ph]]; M. Bleicher and P. Nicolini, J. Phys. Conf. Ser. 237, 012008 (2010) [arXiv:1001.2211 [hep-ph]].

[31] S. W. Hawking, Commun. Math. Phys. 43, 199-220 (1975).

[32] J. D. Bekenstein, Phys. Rev. D 7, 2333 (1973); J. D. Bekenstein, Stud. Hist. Philos. Mod. Phys. 32, 511-524 (2001). [gr-qc/0009019].

[33] R. Emparan, G. T. Horowitz and R. C. Myers, Phys. Rev. Lett. 85, 499 (2000) [arXiv:hep-th/0003118].

[34] S. Chatrchyan et al. [CMS Collaboration], arXiv:1206.5663 [hep-ex]. 